Croyances et perceptions de l'environnement chez les populations riveraines du Parc du haut Niger en Guinée

\title{
Marcel Sow
}

\section{CpenEdition}

\section{Journals}

Édition électronique

URL : https://journals.openedition.org/ere/7298

DOI : $10.4000 /$ ere.7298

ISSN : 2561-2271

Éditeur

Centr'ERE

Référence électronique

Marcel Sow, «Croyances et perceptions de l'environnement chez les populations riveraines du Parc du haut Niger en Guinée », Éducation relative à l'environnement [En ligne], Volume 1 | 1999, mis en ligne le 15 septembre 1999, consulté le 28 mai 2021. URL : http://journals.openedition.org/ere/7298 ; DOI : https://doi.org/10.4000/ere.7298 


\title{
Croyances et perceptions de l'environnement chez les populations riveraines du Parc du haut Niger en Guinée
}

\author{
Marcel Sow
}

1 L'étude des représentations sociales s'avère des plus importantes en éducation relative à l'environnement (ERE) en raison du lien dialectique qu'elles entretiennent avec l'agir. La recherche qui suit souligne l'importance du diagnostic des croyances et des perceptions, comme éléments de représentation, au début de la planification d'un projet d'intervention ou de gestion environnementale. Un tel diagnostic permet d'éclairer la prise de décision quant aux stratégies d'ERE à associer à un plan de gestion intégrée.

\section{Contexte}

2 Situé en Guinée, le Parc national du Haut Niger s'insère dans l'ensemble des projets d'aménagement des bassins versants du Haut Niger et de la Haute Gambie contre la désertification. Du point de vue écologique, le parc renferme un important potentiel de ressources naturelles aux valeurs biologiques, culturelles et économiques insoupçonnées (Projet Parc du Haut Niger, 1998 a, p. 4-5) pouvant contribuer au développement régional et apporter des avantages aux communautés locales. Pour atteindre cet objectif, l'instauration d'un partenariat entre le personnel du parc et les

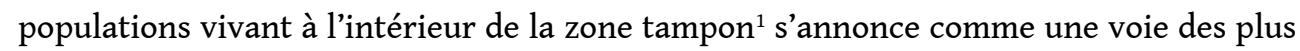
prometteuses. En effet, de nombreuses recherches relatives à la gestion des ressources naturelles ont insisté sur la nécessité de prendre en compte la dimension sociale et sur les avantages qui découlent de la responsabilisation des populations locales. Lusigi (cité dans Projet Parc du Haut Niger, 1998a, p. 53) souligne : 
En Afrique, la croissance démographique est sans doute le facteur qui a l'impact majeur sur les aires protégées. Quatre-vingts pour cent de la population est rurale et elle exerce une forte pression sur toutes les aires, protégées ou non. Pour ce motif, il est fondamental que le parc et les populations puissent partager les objectifs de protection et de gestion des ressources naturelles avec les connaissances à leur disposition.

Sur ce point, Galwen (1992, p. 22) observe «qu'en raison du caractère restrictif des mesures de conservation dans une aire protégée, il est indispensable d'impliquer systématiquement les populations locales riveraines, intéressées au premier chef par le maintien de l'équilibre biologique ». L'auteur insiste particulièrement sur la nécessité d'une intégration des actions de conservation au tissu socioculturel et économique et propose deux avenues de partenariat: d'une part, l'association des populations et des responsables locaux pour la promotion de la conservation des richesses locales et d'autre part, l'implication des acteurs locaux dans le processus de conservation par une valorisation économique.

4 C'est dans cette perspective que se situent les préoccupations majeures du projet Parc du Haut Niger qui, depuis sa genèse en 1994, a mis en place des mesures allant dans le sens de l'implication des populations dans la gestion des aires protégées. En misant sur la structure sociale existante, le parc a favorisé l'émergence de règles au sein des associations traditionnelles ${ }^{2}$ qui reflètent les aspirations des communautés et auxquelles tous les pouvoirs sont légués pour veiller jalousement au patrimoine forestier. Une telle disposition trouve bien sa justification dans une région qui compte plus de 143 villages et hameaux et où la zone intégralement protégée concerne moins de $10 \%$ de la surface totale de cette région.

5 La présente recherche a été réalisée au cours du mois de septembre 1998 dans la région du Parc du Haut Niger. Elle ne représente qu'un volet d'une recherche plus globale au sein du dit parc.

\section{Objectif de recherche et démarche méthodologique}

6 L'objectif de cette recherche était de cerner les croyances et les perceptions des populations de la zone tampon du parc relativement à l'existence du parc et la gestion des ressources naturelles, en vue de bâtir un programme d'éducation relative à l'environnement.

7 Nous avons fait une analyse de documents se rapportant à la problématique de la gestion des ressources naturelles en général et de l'éducation environnementale en particulier. L'avantage de ces données, tel que signalé par Fortin et coll. (cité dans Dionne, 1997, p. 76), "c'est d'être la plupart du temps disponibles et facilement accessibles et surtout de permettre de valider les données en les comparant entre elles ».

8 Pour huit villages localisés dans la zone tampon et appartenant à trois préfectures (Faranah, Dabola et Kouroussa) situées dans la zone d'intervention du parc, nous avons identifié un certain nombre d'acteurs qui interviennent dans la sensibilisation des populations vivant dans la zone tampon. Pour chacun des villages, selon les circonstances, nous nous sommes entretenus avec 4 à 5 interlocuteurs : un Douti, un à deux Wa ton, un Donso ton et un représentant du district. Le choix de ces personnes n'est pas fortuit puisque ces dernières font partie des structures traditionnelles et jouissent 
d'une reconnaissance juridique de la part des autorités du parc. Les entrevues, qui ont pris environ une heure à une heure trente, étaient centrées d'une part, sur les perceptions, les croyances, les attitudes et les comportements actuels des populations en ce qui a trait à la présence du projet du parc dans leurs localités et d'autre part, sur la pratique de l'éducation relative à l'environnement dans leurs milieux respectifs.

\section{Résultats de recherche}

Le parc est perçu comme une zone limitée, un espace de développement social, un lieu de réglementation de la brousse. Il s'agit là de perceptions que nous avons relevées notamment chez les leaders d'opinion de Kouman-di koura (Faranah) et de Saraya (Dabola). Le parc est un service qui donne des «ordres aux villageois » en vue de la préservation des ressources forestières: "Nous veillons au respect de la réglementation parce que les agents du parc nous le recommandent vivement » affirme un Wa ton à Sidakoro. C'est aussi une opinion presque commune qui se dégage du discours de maints leaders d'opinion. Cette observation avait déjà été signalée par le rapport d'activité du 1er semestre 1998 (Projet Parc du Haut Niger, $1998 c$, p. 8), "l'habitant, dans sa vision des choses, a tendance à réduire le parc à un système d'interdiction; tandis que la perspective du parc est une perspective de longue durée, celle de l'habitant est une perspective de moyen terme ».

Nous avons noté que les populations sont en général conscientes de l'existence du parc, mais ne connaissent pas les raisons de son installation. « Pour nous, la présence du parc dans notre village est un prestige, un honneur pour notre village ", souligne un Douti de Toumania (Faranah).

11 Une autre croyance semble émerger dans le discours des leaders qui donne l'impression que les populations approuvent la philosophie du parc par contrainte et non par conviction: "Nous acceptons la présence du parc parce que la forêt et les villages appartiennent à l'état. C'est la raison pour laquelle nous acceptons les conventions du parc ». De l'avis de plusieurs leaders que nous avons interrogés, la présence du parc n'a d'autre implication pour les communautés que l'appui à la réalisation d'infrastructures de base, notamment des ponts, des mosquées, des écoles. Un tel discours laisse entrevoir des éléments qui joueront sur l'implantation d'un partenariat réel entre les villages riverains et le personnel du parc.

Malgré l'existence d'associations traditionnelles qui ont un droit de regard sur la protection des ressources du terroir, il semble que la population ait une attitude réservée par rapport à l'interdiction de toute exploitation dans la zone intégrale de protection : « Pour nous, il est tout à fait normal que les agents du parc nous autorisent, par moments, à prélever le bois de chauffage dans la zone protégée dans la mesure où nos parents avaient l'habitude d'y récolter quelques fagots de bois ", s'exclame un élu local de Dalafilani (hameau situé dans la zone tampon du parc). Pour cette raison, les populations riveraines semblent ne pas adhérer d'emblée à la notion de protection intégrale des ressources. Cette observation est d'ailleurs confirmée par le rapport d'activité du 2e semestre 1998 du projet (Projet Parc du Haut Niger, 1998 d, p. 24) : " Pour les élus locaux, la plus importante source de revenus demeure l'exploitation des ressources du terroir (qui génère par ailleurs des conflits). À l'origine de cette situation : la pression démographique et la recherche de ressources ». 
13 Paradoxalement, nous avons observé chez les leaders d'opinion interrogés, une absence de liens de causes à effets entre l'exploitation et les processus de dégradation des ressources. Si en effet, les populations riveraines sont conscientes des changements climatiques tributaires de la disparition progressive de certaines ressources, il semble qu'elles éludent la réflexion sur les causes de ces phénomènes: «il est vrai que les pluies se raréfient de plus en plus dans nos villages en période de culture, mais nous pensons que cela dépend de la volonté divine. Raison pour laquelle nous faisons des prières dans les mosquées afin que Dieu nous entende pour changer cette situation ». Cette croyance peut s'expliquer en partie par le fort taux d'analphabétisme observé en milieu rural guinéen (environ $75 \%$ de la population) et par l'utilisation de méthodes d'animation peu participatives ${ }^{3}$.

14 Autre illustration de cette situation: la préservation des ressources forestières ne semble pas être considérée par les populations comme une urgence. Aux yeux des populations, l'existence d'un potentiel relativement important de couvert végétal dans le parc fait qu'une menace de dégradation n'est pas perçue comme prioritaire ou même existante. Cette perception est fort répandue dans les localités situées dans la zone tampon: «Nous vivons ici parce que Dieu nous a donné de l'eau, des arbres et des terres fertiles pour subvenir à nos besoins. Donc ce sont des ressources qui demeureront à notre disposition tant que Dieu le voudra " soutiennent les sages dépositaires des traditions orales. Cette conviction, sans doute liée à certaines croyances religieuses et, ou ancestrales, fait que les populations continuent malheureusement à pratiquer l'agriculture extensive dans certaines régions montagneuses malgré un faible rendement et une dégradation de l'environnement.

De ce qui précède, nous retenons que les populations sont conscientes de l'existence du parc, ne serait-ce qu'en raison de la présence de zones interdites d'exploitation et du rôle joué par les structures traditionnelles chargées de gérer les ressources. Toutefois, leur discours suggère qu'elles ne semblent pas considérer ces ressources comme leurs propriétés exclusives. Il y a, semble-t-il, un sentiment de dépendance et de subordination des populations vis-à-vis du parc.

\section{Conclusion et recommandations}

16 À la lumière des observations que nous venons de faire, il nous semble que pour mieux responsabiliser les populations riveraines du parc, une campagne de sensibilisation et d'éducation, via des stratégies de communication comme les animations communautaires et la radio rurale, est un passage obligatoire. L'efficacité d'une telle campagne se mesurera à l'adhésion des populations aux changements proposés. Cela implique un dialogue, une négociation avec les populations afin de trouver les solutions les plus appropriées et les mieux adaptées aux conditions du milieu.

17 En ce qui concerne les animations communautaires, des campagnes d'animation bien ficelées pourraient amener les populations à pratiquer le concept de "forêts villageoises ", forêts entièrement gérées par les populations. Le recours à la méthode GRAAP (Groupe de recherche et d'action pour l'autopromotion paysanne) apparait fort intéressant. À ses débuts, cette méthode a été expérimentée surtout au Burkina Faso. Selon la FAO (1995, p. 64):

Cette méthode, qui regroupe plusieurs outils et techniques de communication, vise à susciter la réflexion et la prise de décision par les populations [...] Elle exploite des 
supports qui facilitent l'approche communautaire villageoise, l'analyse en commun des problèmes environnementaux rencontrés et la recherche de solutions propres au milieu et réalisables localement. documentaires vidéo relatifs à la gestion des ressources naturelles. Ces documents présenteront une série d'images tournées dans le milieu. Ces images non commentées et projetées en une ou plusieurs séances, selon les circonstances, déclencheront des discussions qui amèneront les villageois à voir en face la situation à laquelle ils sont confrontés, à réfléchir sur cette situation et à agir pour améliorer cette situation. La stratégie comprend les étapes suivantes : identification des thèmes environnementaux, réalisation des documentaires vidéo, conception du programme de formation des acteurs ${ }^{4}$, exécution du programme de formation, séances d'animation villageoise dans la zone tampon, évaluation de la démarche et suivi.

Enfin, la troisième stratégie prévue est la radio communautaire utilisée comme support pour la diffusion des messages. Un protocole de collaboration sera établi entre les agents du parc et la radio rurale de la localité pour la production et la diffusion des messages en direction des populations riveraines du parc. Dans l'exécution de ce protocole, un certain nombre d'émissions seront produites sous forme de jeux publics, de magazines, de microprogrammes, de dossiers thématiques dans lesquelles un important temps d'antenne sera donné aux leaders d'opinion (Douti, Wa ton, Ta ton et Donso ton) pour sensibiliser les populations sur le bien-fondé de la gestion intégrée des ressources naturelles. La radio locale, qui diffuse des émissions exclusivement dans la langue du terroir, jouit d'une certaine audience, du moins si l'on se fie à cette observation d'un élu local dans un village de la zone tampon: «la radio favorise une transmission directe, une écoute simultanée et évite une perte d'énergie ». La stratégie prévoit les étapes suivantes : établissement d'un protocole de collaboration avec la radio communautaire, identification des thèmes avec les leaders d'opinion pour constituer le corpus du message, conception des messages, diffusion des messages et évaluation de la démarche via une grille d'évaluation qui prendra en compte un certain nombre d'indicateurs comme l'accès du public au message, la rétention du message, l'enrichissement des connaissances, l'attitude du public et la mise en œuvre des conduites préconisées dans les messages. 


\section{BIBLIOGRAPHIE}

Dionne, L. (1997). Conceptions, croyances et pratiques en matière d'éducation à l'environnement chez les éducateurs du primaire en République de Guinée. Mémoire de maîtrise en sciences de l'éducation, Université du Québec à Montréal.

FAO. (1995). Approche participative, communication et gestion des ressources forestières en Afrique sahélienne. Bilans et perspectives. Rome : Édition FAO.

Galwen, J.C. (1992). Gestion participative des aires protégées. Paris : Édition IRAM.

Projet Parc du Haut Niger. (1998a). Projet d'aménagement du Parc du Haut Niger 1999-2003 volume I et volume II. Conakry : Programme Régional d'aménagement des bassins versants du Haut Niger et de la Haute Gambie.

Projet Parc du Haut Niger. (1998 b). Procès-verbal de la troisième assemblée des populations périphériques du Haut Niger, Faranah. Conakry : Programme Régional d'aménagement des bassins versants du Haut Niger et de la Haute Gambie.

Projet Parc du Haut Niger. (1998 c). Rapport d'activité du 1er semestre, Faranah. Conakry : Programme Régional d'aménagement des bassins versants du Haut Niger et de la Haute Gambie.

Projet Parc Haut Niger. (1998 d). Rapport d'activité du 2e semestre, Faranah. Conakry :

Programme Régional d'aménagement des bassins versants du Haut Niger et de la Haute Gambie.

\section{NOTES}

1. Cette zone tampon est caractérisée par des villages relativement grands concentrés le long des axes routiers principaux et des pistes. Ces villages exercent une grande pression sur leur environnement.

2. Il s'agit d'associations composées des Ta ton (surveillants des feux de brousse), Donso ton (associations des chasseurs), Wa ton (ensemble des Ta ton et des Donso ton) et des Douti (représentants des sages du village) (Projet Parc Haut Niger, 1998 b, p. 3).

3. Nous observons que les formes d'animation les plus couramment utilisées sont les réunions avec les populations qui se tiennent de façon épisodique et qui se limitent à de simples comptesrendus au cours desquels les leaders d'opinion se contentent de transmettre les intentions du personnel du parc. Ce genre de rencontres ne fait que renforcer la dépendance des structures traditionnelles vis-à-vis du parc.

4. Dans un premier temps, seuls les huit animateurs de zones seront concernés par ce programme de formation et cela, compte tenu de l'expérience qu'ils ont accumulée et de la complexité de ce mode d'animation. 


\section{AUTEUR}

\section{MARCEL SOW}

Marcel Sow détient une maîtrise en biologie de l'Université Guinéenne et une maîtrise en sciences de l'environnement de l'Université du Québec à Montréal. Il est chargé du programme d'éducation relative à l'environnement au Centre d'étude et de recherche en environnement de l'Université de Conakry. Parallèlement, il s'occupe de divers projets nationaux en matière de préservation des ressources naturelles et réalise des productions médias. 